\title{
Implementing Structured Follow-Up of Neonatal and Paediatric Patients: An Evaluation of Three University Hospital Case Studies Using the Functional Resonance Analysis Method
}

\section{Véronique Bos ( $\nabla$ v.l.bos@amsterdamumc.nl )}

Amsterdam UMC - Locatie AMC: Amsterdam UMC Locatie AMC https://orcid.org/0000-0002-74479662

\section{Daniëlle Roorda}

Emma Childrens' Hospital UMC: Emma Kinderziekenhuis Amsterdam UMC Eleonore de Sonnaville

Emma Childrens' Hospital UMC: Emma Kinderziekenhuis Amsterdam UMC

\section{Menne van Boven}

Emma Childrens' Hospital UMC: Emma Kinderziekenhuis Amsterdam UMC Jaap Oosterlaan

Emma Childrens' Hospital UMC: Emma Kinderziekenhuis Amsterdam UMC Johannes van Goudoever

Emma Childrens' Hospital UMC: Emma Kinderziekenhuis Amsterdam UMC

Niek Klazinga

Amsterdam UMC - Locatie AMC: Amsterdam UMC Locatie AMC

\section{Dionne Kringos}

Amsterdam UMC - Locatie AMC: Amsterdam UMC Locatie AMC

\section{Research article}

Keywords: follow-up, implementation science, quality improvement, long-term outcomes, functional resonance analysis method (FRAM), neonatal intensive care, paediatric intensive care, paediatric surgery

Posted Date: September 3rd, 2021

DOI: https://doi.org/10.21203/rs.3.rs-408416/v2

License: (9) This work is licensed under a Creative Commons Attribution 4.0 International License. Read Full License 
Version of Record: A version of this preprint was published at BMC Health Services Research on February 14th, 2022. See the published version at https://doi.org/10.1186/s12913-022-07537-x. 


\section{Abstract}

Background: In complex neonatal and paediatric clinical practice, little is known about long-term patient outcomes and what follow-up care is most valuable for patients. Emma Children's Hospital, Amsterdam UMC (Netherlands), implemented a follow-up programme called Follow Me for neonatal and paediatric patient groups, to gain more insight into long-term outcomes and to use such outcomes to implement a learning cycle for clinical practice, improve follow-up care and facilitate research. Three departments initiated re-engineering and change processes. Each introduced multidisciplinary approaches to long-term follow-up, including regular standardised check-ups for defined age groups, based on medical indicators, developmental progress, and psychosocial outcomes in patients and their families. This research evaluates the implementation of the three follow-up programmes, comparing predefined procedures (work-as-imagined) with how the programmes were implemented in practice (work-as-done).

Methods: This study was conducted in 2019-2020 in the outpatient settings of the neonatal intensive care, paediatric intensive care and paediatric surgery departments of Emma Children's Hospital. It focused on the organisational structure of the follow-up care. The functional resonance analysis method (FRAM) was applied, using documentary analysis, semi-structured interviews, observations and feedback sessions.

Results: One work-as-imagined model and four work-as-done models were described. The results showed vast data collection on medical, developmental and psychosocial indicators in all work-as-done models; however, process indicators for programme effectiveness and performance were missing. In practice there was a diverse allocation of roles and responsibilities and their interrelations to create a multidisciplinary team; there was no one-size-fits-all. Although control and feedback loops for long-term outcomes were specified with respect to the follow-up groups within the programmes, they were found to overlap and misalign with other internal and external long-term outcome monitoring practices.

Conclusion: Implementing structured long-term follow-up may provide insights for improving daily practice and follow-up care, with the precondition of standardised measurements. Lessons learned from practice are (1) to address fragmentation in data collection and storage, (2) to incorporate the diverse ways to create a multidisciplinary team in practice, and (3) to include timely actionable indicators on programme effectiveness and performance, alongside medical, developmental and psychosocial indicators.

\section{Contributions To The Literature}

- Provides in depth analysis of three re-engineered change processes that incorporate follow-up data collection and feedback cycles on long-term paediatric care outcomes

- Explores the value of the functional resonance analysis method to incorporate learning from practice across departments which have implemented re-engineering and change processes to structure follow-up care and long-term outcomes 
- Exposes gaps found between work-as-imagined and work-as-done in terms of (a) data collection and standardisation of follow-up care and long-term outcomes, (b) key stakeholder roles and responsibilities, and (c) the building of control and feedback loops to aid decision-making.

\section{Background}

To date, data collection in healthcare systems has been predominantly designed to obtain short- and intermediate-term data on treatment outcomes for patients. The standardised collection of long-term outcome data and data on follow-up care is faced with many challenges; it is therefore often absent ( 1 , 2). As pressures increase to maintain and improve the health of populations, the objective of high-value healthcare systems, as described by Porter, has gained importance on national policy agendas (3-5). Supported by Donabedian's model of structure, process and outcome, a standardisation of outcome measurements is needed to gain necessary insights for improvement $(6,7)$.

The field of neonatal and paediatric clinical practice has experienced substantial improvements in shortterm survival and mortality rates (8-11). In light of the higher short-term survival rates, a shift of focus towards long-term outcomes is now in progress, in order to support children with adequate follow-up care. Although regular medical follow-up of patients is already taking place in many patient groups, systematic data collection and standardised protocols for follow-up care are often missing. Follow-up programmes can be used to standardise content in follow-up practices and to systematically collect data on long-term outcomes, in order to provide the insights needed to aid decision-making on treatment and follow-up care $(12,13)$. In rare and complex paediatric patient groups, disease and treatment may have effects on children's development, and advances in treatment are moving at a rapid pace (14). Hence, systematic data collection in rare paediatric patient groups needs to be more comprehensive and to encompass longterm medical, developmental and psychosocial indicators (15-17). For this reason, three separate reengineering and change processes for follow-up were implemented at Emma Children's Hospital, Amsterdam UMC (Netherlands), targeting three high-cost, highly complex patient groups: (1) patients treated in the neonatal intensive care units (NICUs), (2) patients treated in the paediatric intensive care unit (PICU), and (3) patients who had undergone neonatal surgical treatment for congenital malformations (paediatric surgery department).

Re-engineering and change processes in daily clinical practices are known to be challenging. By evaluating the three implemented follow-up programmes, this research aims to learn from daily practice and to improve the quality and sustainability of the follow-up programmes as well as their impact on clinical practice. Our research objective is to evaluate the implementation of the outpatient follow-up programmes of the neonatal ICUs, paediatric ICU and paediatric surgery departments by comparing work in practice (work-as-done), with the mission, vision, organisational blueprint and tools established by the management team of the Follow Me programme (work-as-imagined). The following questions are addressed: (1) How is the implemented follow-up programme (a) generating data collection and standardisation for the measurement of long-term outcomes, (b) allocating key stakeholder roles and responsibilities, and (c) building control and feedback loops to aid decision-making? (2) Can gaps 
between work-as-imagined and work-as-done be identified in the three case studies and, if so, how could such gaps be overcome using within- and cross-case-study learning?

\section{Methods}

The study conforms to the Consolidated Criteria for Reporting Qualitative Studies (COREQ, Additional file 1).

\section{Setting}

The work-as-imagined model is based on the Follow Me programme of Emma Children's Hospital, Amsterdam UMC. The four work-as-done models are based on the observed outpatient settings connected to the two neonatal ICUs, the paediatric ICU and the paediatric surgery department of Emma Children's Hospital (see Table 1 for case study descriptions). As a result of a recent merger between two university medical centres, neonatal intensive care still had two separate post-ICU outpatient clinics, at the locations Academic Medical Centre (AMC) and VU University Medical Centre (VUMC); work-as-done for neonatal intensive care was therefore studied at both locations.

Table 1 Case study descriptions

\section{Research design}

The functional resonance analysis method (FRAM) was applied. Documentary analysis, semi-structured interviews, observations and feedback sessions were conducted to identify key functions and their aspects and interrelations, enabling us to model and compare work-as-imagined (predefined procedures) with work-as-done. The scope of the models was the organisational context of the follow-up programme in terms of (a) generating data collection and standardisation for the measurement of long-term outcomes, (b) allocating key stakeholder roles and responsibilities, and (c) building control and feedback loops to aid decision-making. Close collaboration between researchers and the staff participating in the follow-up programmes was established in order to tailor the research to the contexts of the case studies, to accurately model the results, and to stimulate mutual learning and improvement by capitalising on experiences from practice and on research findings.

\section{The functional resonance analysis method (FRAM)}

The FRAM method is described by Hollnagel and colleagues $(18,19)$. It is a systematic, participatory approach designed to model how complex processes usually go right in practice (work-as-done) and to compare work-as-done with predefined procedures (work-as-imagined) (20-23). FRAM models the workas-done by pinpointing relevant functions. Functions are defined as 'the activities - or set of activities that are required to produce a certain outcome' (18). Functions are displayed in hexagons (Figure 1) and 
then described using six aspects: Input (I), Output (O), Preconditions (P), Resources (R), Control (C) and Time (T) (Table 2). Potential couplings between functions are added by identifying shared attributes of aspects. Such couplings represent possible relationships or dependencies between functions, but do not refer to any particular situation. Not all aspects of each function need couplings. Couplings are generally $n$-to- $n$ rather than 1-to-1 (22).

Table 2 Aspects of FRAM functions

\section{Data collection and coding}

Our data collection took place from September 2019 to July 2020. To gather data on the work-asimagined, we collected internal protocols and management documents on the follow-up processes ( $n=$ $16)$ and composed and conducted semi-structured interviews $(n=10)$ with all members of the overarching project team of the Follow Me programme $(n=4)$, with the responsible departmental manager per case study or department $(n=3)$, and with the responsible coordinator for the Follow $\mathrm{Me}$ programme per case study or department $(n=3)$. The interviewees gave informed consent to the research, and interviews were audio-recorded. To obtain relevant data from the interviews, we used as an interview guide the set of standard questions from the FRAM methodology as proposed by Hollnagel and colleagues (19). To gather data on the work-as-done, we conducted at least 2 observations of full outpatient follow-up visits for every case study (total $n=8$ ), studied work documents on outcomes of the follow-up programmes $(n=2)$, and conducted observations during an evaluation session on outcomes per case study, if held during the study period $(n=2)$. Informed consent to the research was obtained from the patients and their families, and from health care providers involved in the observations, before the start of the outpatient follow-up visits. A template for note-taking was used during observations, focused on identifying functions, aspects and interdependencies (see template in Additional file 2). An overview of collected data and data use per model can be found in Additional file 3 .

\section{Modelling and calibration of models}

The first author coded the collected data into functions, in line with the Hollnagel handbook (19). Related aspects (Input, Output, Preconditions, Resources, Control and Time) were added to the functions, and potential couplings were drawn. The list of identified functions, aspects and potential couplings per model was then scanned to identify those functions and aspects that affected the three organisational scope themes (see a, b and c above in section research design). If one such theme applied to a function or aspect, that function or aspect was defined as a foreground function or aspect and shown in the models. Background functions judged as not having that potential were not shown in the models. A total of five models were constructed: one work-as-imagined model for the Amsterdam UMC Follow Me programme and four work-as-done models, two for the neonatal ICUs (Locations AMC and VUMC), one for the paediatric ICU and one for the paediatric surgery department. The FRAM Model Visualizer (FMV) software, version 2.1.0, was used to shape the models (24). 


\section{Analysis of the models}

A descriptive analysis of the models was made, which defined key functions, aspects and potential couplings for the three organisational scope themes ( $a, b$ and $c$ ). FRAM improvement sessions per department $(n=3)$ were organised to validate findings. Only one content change was made to the models following those sessions: a separation of patient-reported outcome measurement (PROM) routes for (1) psychosocial indicators and (2) medical indicators in the paediatric surgery model. Some visual changes were also made, such as placement of key stakeholders separately in the model to highlight them.

\section{Learning within and across case studies}

The descriptive analysis findings were used to identify gaps between the work-as-imagined and work-asdone models in relation to the three organisational scope themes ( $a, b$ and $c)$ both within and across case studies. Improvement sessions with each department were organised to discuss the findings and highlight areas for improvement per department and for the Follow Me programme as a whole.

\section{Results}

\section{Work-as-imagined model in the Follow Me programme}

In the analysis of work-as-imagined in the Follow Me programme, 18 functions were identified, of which 8 were selected as foreground functions (Figure 2).

\section{Data collection and standardisation}

Standardisations of data measurement instruments were considered to be Preconditions for evaluating outcomes (function 5 in Figure 2) and for conducting research (function 6 in Figure 2). In turn they were to enable creation of a knowledge base on disease, treatment and follow-up outcomes. The functions that generated data collection and their aspects are shown in Additional file 4.

\section{Allocation of key stakeholder roles and responsibilities}

The key stakeholder in the work-as-imagined model was the patient, surrounded by a multidisciplinary team providing standardised follow-up care and personalised care. Patient organisations were to have a Control, or monitoring, aspect in maintaining a patient-oriented approach. Academic centres had a Control aspect in benchmarking treatment outcomes and were seen as learning partners in these complex, often rare, patient groups. A dedicated project team for the Follow Me programme initiated outcome evaluation sessions. 


\section{Building control and feedback loops}

The functions (5) to evaluate outcomes, (6) to conduct research, (7) to standardise treatment protocols and (8) to standardise the follow-up programme were to be used to build control and feedback loops designed to improve treatment and follow-up protocols. Preconditions within feedback loops were the systematic collection of standardised PROMs and the performance of standardised measurements and data collection on medical, developmental and psychosocial outcomes by the multidisciplinary team during the follow-up. To establish and continuously improve follow-up protocols, the standardisation process of the follow-up programme was to make use of research output, evaluations of follow-up outcomes, the experience of the multidisciplinary team, and the cooperative relationships with patient organisations and with other academic centres. Such interrelations formed a cycle of collection of longterm outcomes, and these were to feed into the insights that would provide feedback on treatment and follow-up protocols.

\section{Work-as-done at the neonatal ICU, Location AMC}

In the analysis for the work-as-done model of the AMC neonatal ICU, 12 functions were identified, of which 10 were selected to be foreground functions (Figure 3).

\section{Data collection and standardisation}

Standardisation of data measurement instruments, including standardised data collection time points, was based on national guidelines in place since the 1990s (12). The functions that generated data collection and their aspects are shown in Additional file 4.

\section{Allocation of key stakeholder roles and responsibilities}

A patient defined by the national guidelines as belonging to an at-risk group was offered the standardised follow-up programme in two parts, one with the neonatologist and one with the psychologist. There was an alignment between the neonatologist and the psychologist, with their functions interlinked by consecutive time slots and patient handover. A patient could receive additional or adjusted care, referral or guidance from both the neonatologist and the psychologist individually.

\section{Building control and feedback loops}

In this model, small feedback loops were seen that monitored previous functions or Preconditions for downstream functions. In addition, the face-to-face handover between the neonatologist and the psychologist incorporated a Control aspect to make sure a consent form for data use for research purposes had been signed and collected (a Precondition for conducting research). Standardised regular 
internal evaluation sessions on the outcomes of follow-ups did not take place at the AMC neonatal ICU at the time of this research, and were therefore not modelled.

\section{Work-as-done at the neonatal ICU, Location VUMC}

The analysis for the work-as-done model of the neonatal ICU, Location VUMC, included 17 functions, of which 13 were defined as foreground functions (Figure 4).

\section{Data collection and standardisation}

Standardisation of data measurement instruments, including standardised data collection time points, was based on national guidelines in place since the 1990s (12). All the health care providers involved prepared the follow-up separately, but were aided by a summary of the patient's history constructed by a medical psychology trainee with the help of a template. That summary was only available to all the involved health care providers if cognitive development was also assessed (not done in all age groups). The functions that generated data collection and their aspects are shown in Additional file 4.

\section{Allocation of key stakeholder roles and responsibilities}

Patients defined by the national guidelines as belonging to an at-risk group were offered the standardised follow-up care involving four consultations. The first was with the psychologist (if the patient was in the age group offered assessment of cognitive development) in a separate but nearby outpatient area. Subsequent sessions were planned with the medical assistant, the neonatologist and the physiotherapist in another outpatient area. All consultations were scheduled consecutively, thus minimising patient waiting time. The follow-up care was coordinated by a designated medical assistant with a host function towards the patient. The assistant welcomed the patient and provided guidance on which questions to pose to which health care provider. In case the next planned provider was not ready to conduct the followup when the patient was available, the assistant tried to switch around consultations, thus reducing delays for the patient and providers alike. The assistant also provided brief patient handovers in person to the health care providers, highlighting certain concerns the patient might have and giving an update on consultations so far. The role and responsibilities of the host function were confined to the follow-up programme within the department. In our observations, patients who had received treatment in two different academic neonatal ICUs during hospital stays would now be attending two similar follow-up programmes, one in each academic centre, as both centres were compliant with national guidelines. There was a stronger interlinkage between the neonatologist and the physiotherapy disciplines; those functions were partially performed simultaneously in the same room. This facilitated communication between the two professions and allowed for the parents of the patient to be questioned by the intensivist, while the physiotherapist observed the infant or child in terms of motor development. 


\section{Building control and feedback loops}

A Control function was created that reminded the patient of the follow-up and the PROMs questionnaire to be completed two weeks beforehand. The prepared summary and the preparation functions for each follow-up function involving health care professionals (functions 6, 9, 10 and 11) served as Controls for the data availability needed for the follow-up programme. Individual results from the professionals' follow-ups were discussed in a multidisciplinary meeting straight after the follow-ups to align thoughts on future treatment. Standardised regular internal evaluation sessions on the outcomes of follow-ups were not being conducted in the VUMC neonatal ICU department at the time of this research, and were therefore not modelled.

\section{Work-as-done in the paediatric ICU follow-up}

The analysis for the paediatric ICU's work-as-done model included 11 functions, of which 8 were defined as foreground functions (Figure 5).

\section{Data collection and standardisation}

Standardisation of the follow-up programme was based on the national guidelines for follow-up after paediatric intensive care (13). Patients had problems with delivering the patient-reported outcomes in time for the follow-up programme; in one of our observations, four out of four patient families had failed to meet that goal. That resulted in fewer Resources for the psychologist to prepare for the follow-up and could lead to inadequate Resources or bias for outcome evaluation and research. In our observations, patients reported receiving the invitation to the online portal for the PROM questionnaires on paper, whereas they preferred digital invites to access the questionnaires directly by clicking on a link whilst at the computer. Another observed problem was confusion in a patient's parents about which questionnaire was needed for the current follow-up; they referred to other evaluation questionnaires received after the hospitalisation and a questionnaire from a children's support service outside the scope of the department. All data on medical indicators were collected using standardised templates in the patient EHR. The functions that generated data collection and their aspects are shown in Additional file 4.

\section{Allocation of key stakeholder roles and responsibilities}

The modelled work-as-done started during the discharge procedure from the paediatric intensive care unit. With the help of a decision tree, a decision was made by a paediatrician-in-training, supervised by a paediatric intensivist. In addition to patients included in line with the decision tree, other patients might be included in the follow-up programme by exception on advice of clinicians and nurses who had treated the patient. If a positive decision was made, the patient was offered the standardised follow-up in three parts, first seeing the medical assistant of the outpatient clinic, then the paediatric intensivist, and then the 
psychologist. If necessary, a paediatric pulmonologist, cardiologist, neurologist or neuropsychologist could be included in the follow-up, but such consultations were not needed for the patients we observed. Key stakeholders were the paediatric intensive care specialist and the psychologist. The interlinkage between the functions of these stakeholders was reinforced by close proximity, consecutive time slots and face-to-face patient handovers. In our observations, however, a face-to-face handover was not always possible due to misalignments of available time caused by delays in consultations.

\section{Building control and feedback loops}

Both the paediatric intensivist and the psychologist prepared for the follow-up in order to ensure Preconditions for the physical follow-up with the patient. No Time aspect interlinked these functions, however; hence, if necessary information, such as PROM results, was found to be missing, there was no opportunity to rectify that in time for the follow-up consultations. One patient in our observations had undergone a lung function test the week before the follow-up, but the test results from the pulmonologist were scheduled for after the follow-up clinic visit. This suggests possible misalignment between the follow-up programme and routine medical check-ups. The observations detected no Control function to check alignment with routine medical follow-up procedures. The Follow Me programme in the paediatric ICU convened a yearly improvement session, attended by all the participating health care providers, patient organisations and the departmental management team, to evaluate outcomes, protocols and organisational indicators.

\section{Work-as-done model in the paediatric surgery follow-up}

The analysis for the work-as-done model in the paediatric surgery department's outpatient clinic identified 15 functions, of which 12 were defined as foreground functions (Figure 6).

\section{Data collection and standardisation}

The time spent between patient and health care providers was largely being used for data collection purposes. One family reported during our observations that it was not clear to them whether PROMs about parental psychological outcomes were available to specific health care providers; as the PROMs included personal information on psychosocial factors involving the parents, they found it odd not to know who was aware of that information. PROMs on psychosocial factors were in fact reviewed by the psychologist only, who drew on them to provide input in multidisciplinary meetings after the outpatient visit. Other health care providers did have access to the PROMs before the outpatient care began, but, in our observations, use was not made of that access. The functions that generated data collection and their aspects are shown in Additional file 4. 


\section{Allocating key stakeholder roles and responsibilities}

The standardised follow-up of the paediatric surgery department could consist of up to seven parts (Figure 6, functions 3 to 9), depending on the patient's age, disease or treatment. The multidisciplinary meeting between health care professionals that took place after the physical follow-up (not in the patient's presence) was the medium used to exchange professional viewpoints. Interlinkages between professionals whilst a patient was present at the outpatient clinic were few. One interlinkage was observed between the clinical nurse specialist and the paediatric surgeon via brief patient handovers, or when that nurse was physically present while the paediatric surgeon was in consultation with the patient. The order in which professionals saw a patient was not standardised, but was determined by the most efficient schedule. Thus, health care providers involved in the follow-up would see their patients at different stages of the follow-up (they might be the first, second, third or last to see the patient). What previous functions had been performed might be obvious from a patient's perspective, but complicated to oversee for each individually involved health care provider, even though a schedule for the day was accessible to all involved. In the post-surgery work-as-done, individual care needs of patients and their families were addressed in fragmented ways, with each care provider discussing important medical observations for their field independently. If an aberration in one function occurred, it was dealt with mostly within the discipline in question.

\section{Building control and feedback loops}

Few Control aspects were observed in the surgery work-as-done model. Health care providers were guided by standardised templates in a patient's electronic health record (EHR). The Follow Me programme of the paediatric surgery department convened a yearly improvement session, attended by all the participating health care providers, patient organisations and the departmental management team, to evaluate outcomes, protocols and organisational indicators.

\section{Gaps between work-as-imagined and work-as-done models; learning across case studies}

In all four work-as-done models, the implemented data collection was aided by templates in the patient EHRs (such as medical assessments) or by standard measurement instruments (such as PROM questionnaires or Bayley Scales of Infant Development), using multiple entry points, EHR modules and software programs. Varying by case studies, the choices regarding data collection on follow-up care mirrored the provision of 'standard' care, with the follow-up model of the paediatric surgery department being more specialised and more fragmented, whereas that of the paediatric ICU was more encompassing and less fragmented. 
In all four case studies, data were collected and evaluated on medical, developmental and psychosocial outcomes; however, data collection and reporting on the effectiveness of the follow-up was limited. Indicators were missing, for example, to show the number of patients seen for follow-up as a proportion of those that should have been seen according to national guidelines or internal protocols. Also missing were indicators providing insights into changes made to treatment plans due to the follow-up programme. Process indicators on the follow-up programme in the studied departments were limited to the number of no-shows (highlighting efficiency in planning and success in informing patients about the importance of the programme); they were not available on the effectiveness of the follow-up programme.

In practice, implementation of a multidisciplinary team approach came in many forms, including (1) a predetermined consecutive order of consultation for the care providers involved (e.g. AMC neonatal ICU and paediatric ICU); (2) access to multiple interdisciplinary data collection points by an individual provider (e.g. paediatric ICU, neonatologist); (3) team-up by providers for data collection (e.g. physiotherapist and neonatologist together in one consultation at the VUMC neonatal ICU); (4) face-toface patient handovers by paired care providers (neonatologist and psychologist at the paediatric ICU); or (5) a hosting function created to facilitate patient handovers (e.g. VUMC neonatal ICU). Responsibilities for coordinating the follow-up were allocated in different ways in the multidisciplinary teams, for example via the medical assistant (VUMC neonatal ICU), via the lead clinician (AMC neonatal ICU and paediatric $\mathrm{ICU})$ or via the clinical nurse specialist (paediatric surgery).

Yearly evaluation sessions to improve treatment and follow-up, and multiple small control loops to check whether Preconditions were being met, were present in most work-as-done models. However, poor timeliness of control and feedback loops and the lack of process indicators limited the ability of programmes to make adjustments when discrepancies were found. In practice, control and feedback loops operated solely within the scope of the follow-up programmes. This resulted in unplanned interactions with 'routine care' outside the department or organisation in almost all work-as-done models (VUMC neonatal ICU, paediatric ICU, paediatric surgery).

\section{Discussion}

This study used the FRAM methodology to evaluate the implementation and organisation of dedicated neonatal and paediatric follow-up care programmes by comparing work-as-done with work-as-imagined. First, our findings revealed an emphasis on medical, developmental and psychosocial data collection. We believe follow-up programmes can benefit from adding process indicators in order to respond in a timely fashion to discrepancies in the follow-up care and to adequately assess effectiveness and performance in the programmes. As it is still largely unknown what follow-up care is most valuable to patient groups in paediatric care, it is essential that the effectiveness of follow-up programmes be readily monitored (25). Previous research has shown the potential of process indicators in situations where outcome indicators lack in actionability $(6,26)$. Second, our findings have shown that multidisciplinary teams can take on 
many forms in practice and that the needs of multidisciplinary teams may differ in different settings. We acknowledge previous research on the importance of incorporating the real-world context (and its people) (27) in order to successfully implement new programmes and advise programme adjustments according to the context and needs of health care professionals and patients.

Our findings also show that the organisation of follow-up care is still based on academic specialisations and superspecialisations which may not align with the broader health, developmental and psychosocial monitoring needed to achieve satisfactory long-term outcomes for paediatric patients and their families $(28,29)$. Data collection remains fragmented per medical specialisation, diagnosis or treatment group. The same is seen in the Dutch medical quality registers. Every quality register has been initiated with the respective subinterests in mind, and they contain few interlinkages to other health and social care databases (30). As treatments and follow-up care by multiple health, social and developmental care providers interrelate with child environmental factors, comprehensive data infrastructures can be important tools for adequately monitoring long-term outcomes.

The measurement of healthcare performance, and the use of healthcare performance data by different end-users (such as providers or patients), are important strategies to improve our health care services and systems in line with the Triple Aim programme for health care change (better health, better patient experience, cost control) (31). However, responsibilities for long-term patient outcome assessment and follow-up do not appear to be clearly allocated. Further research is needed to understand how follow-up Outcomes can be adjusted to better address the patient perspective and the patients' broader health, psychosocial and developmental outcomes, instead of mirroring the organisational structures of health care provision.

\section{Applicability of the FRAM method}

The FRAM method required special training and close collaboration between research and parties in practice. This resulted in the strengths and weaknesses listed below. Using direct observations, we were able to view the real-world context by studying the work-as-done, an often overlooked and underreported perspective in research (32).

\section{Strengths, difficulties and limitations}

A strength of this study was the close collaboration between researchers, the Follow Me project team and the coordinators of the follow-up programmes, resulting in a multidisciplinary, practice-oriented research team. Use of the FRAM methodology with multiple departments implementing similar programmes created cross-case-study learning opportunities. Difficulties in this study included the incorporation of FRAM models into the reflection sessions with the departments, as the methodology was new to most and required some effort to explain. The author VB was trained in the methodology and led the sessions; topics addressed by discussion participants were bountiful and relevant. A limitation of the FRAM method in our study lay in the difference in details observed for the work-as-imagined versus the work-asdone models, although that is an observation on its own. Finally, in order to keep the models readable, our 
necessary choices regarding foreground and background functions forced us to reflect a limited scope of the 'real world'.

\section{Conclusion}

The follow-up programmes of the three neonatal and paediatric patient groups showed vast data collection practices for medical, developmental and psychosocial indicators, a diverse allocation of roles and responsibilities to create multidisciplinary teams, and many small control and feedback loops.

Gaps to overcome are to address overspecialisation in data collection, storage and infrastructures; to incorporate and facilitate diversity in multidisciplinary teams in practice; and to include timely actionable indicators to measure effectiveness and performance in the follow-up programme itself. The FRAM methodology enabled a structured reflection on the progress of the implementation, based on the observed differences between work-as-imagined and work-as-done in the three teams as well as across the teams.

\section{Abbreviations}

AMC Academic Medical Centre

EHR electronic health record

FRAM functional resonance analysis method

ICU intensive care unit

LNF Landelijke Neonatale Follow-up

NICU neonatal intensive care unit

NVK Nederlandse Vereniging voor Kindergeneeskunde (Dutch Paediatric Society)

PICU paediatric intensive care unit

PROM patient-reported outcome measurement

UMC University Medical Centre

VUMC VU University Medical Centre Amsterdam

\section{Declarations}

\section{Ethics approval and consent to participate}


This research proposal was reviewed and approved prior to data collection by the Medical Ethics Review Committee of the Academic Medical Centre, Amsterdam (reference number W19_325 \# 19.384 app.). Informed consent was obtained from all interviewees and all health care providers, patients and their families included in the observations. All methods were carried out in accordance with relevant guidelines and regulations.

\section{Consent for publication}

This manuscript does not contain any individual person's data in any form. Written consent for publication of the Follow Me case study on neonatal and paediatric intensive care and paediatric surgery was granted by the project leadership of the Follow Me programmes of the Amsterdam UMC and is available upon request.

\section{Availability of data and materials}

The digitised data collected and analysed is stored at the Amsterdam UMC and is available upon reasonable request via the corresponding author.

\section{Competing interests}

The authors VB, DK and NK declare no competing interests. The authors DR, ES, MB and JO declare competing interest due to responsibility for Follow Me programme implementation in the Amsterdam UMC. The author JG declares a competing interest as the initiator of the UMC's Follow Me programme.

\section{Funding}

The Amsterdam Medical Centre funded the PhD grant for the researcher VB. The funding body had no role in the design of the study and the collection, analysis and interpretation of the data, nor in the writing of the manuscript.

\section{Authors' contributions}

The research proposal was drafted by VB, DR, ES, MB, JO, NK and DK and executed by VB under close collaboration and supervision of DK and NK. DR, ES and MB provided communication and planning with the departments. The manuscript was written by VB under close collaboration and supervision of DK and NK; it was peer-reviewed multiple times by DR, ES, MB, JO and JG. All authors have read and approved the manuscript. 


\section{Acknowledgements}

The authors thank all the patient and their families and health care professionals who were willing to reflect so openly on the process during the observations, interviews and team discussions.

\section{References}

1. O'Connor DP, Brinker MR. Challenges in outcome measurement: clinical research perspective. Clin Orthop Relat Res. 2013;471(11):3496-503.

2. Wilcox ME, Ely EW. Challenges in conducting long-term outcomes studies in critical care. Curr Opin Crit Care. 2019;25(5):473-88.

3. Wouterse B, Smid B. How to finance the rising costs of long-term care: Four alternatives for the Netherlands. Fiscal Studies. 2017;38(3):369-91.

4. Kluge H. A new vision for WHO's European Region: united action for better health. The Lancet Public Health. 2020;5(3):e133-e4.

5. Porter ME. What is value in health care? N Engl J Med. 2010;363(26):2477-81.

6. Donabedian A. Evaluating the quality of medical care. 1966. Milbank Q. 2005;83(4):691-729.

7. Donabedian A. The quality of care. How can it be assessed? Jama. 1988;260(12):1743-8.

8. Costeloe KL, Hennessy EM, Haider S, Stacey F, Marlow N, Draper ES. Short term outcomes after extreme preterm birth in England: comparison of two birth cohorts in 1995 and 2006 (the EPICure studies). Bmj. 2012;345:e7976.

9. Namachivayam P, Shann F, Shekerdemian L, Taylor A, van Sloten I, Delzoppo C, et al. Three decades of pediatric intensive care: Who was admitted, what happened in intensive care, and what happened afterward. Pediatr Crit Care Med. 2010;11(5):549-55.

10. Epstein D, Brill JE. A history of pediatric critical care medicine. Pediatr Res. 2005;58(5):987-96.

11. Matsumoto N, Hatachi T, Inata Y, Shimizu Y, Takeuchi M. Long-term mortality and functional outcome after prolonged paediatric intensive care unit stay. Eur J Pediatr. 2019;178(2):155-60.

12. Follow-up WLN. Aanbeveling Landelijke Neonatale Follow-up - NICU follow-up. 2015.

13. Kindergeneeskunde NVv. Richtlijn Follow-up van kinderen na opname op een intensive care. 2017.

14. Drotar D. Measuring health-related quality of life in children and adolescents: Implications for research and practice. Psychology Press; 2014.

15. Taylor A, Butt $W$. The evaluation of outcome following paediatric intensive care: the major issues identified. Clinical Intensive Care. 2000;11(5):239-44.

16. Johnson S, Marlow N. Early and long-term outcome of infants born extremely preterm. Arch Dis Child. 2017;102(1):97-102.

17. Porter ME, Pabo EA, Lee TH. Redesigning primary care: a strategic vision to improve value by organizing around patients' needs. Health Aff (Millwood). 2013;32(3):516-25. 
18. Hollnagel EFRAM. The Functional Resonance Analysis Method: Modelling Complex Socio-Technical Systems2012.

19. Hollnagel E, Hounsgaard J, Colligan L. FRAM - the Functional Resonance Analysis Method - a handbook for the practical use of the method. Denmark: Centre for Quality; 2014.

20. Damen NL, de Vos MS, Moesker MJ, Braithwaite J, de Lind van Wijngaarden RAF, Kaplan J, et al. Preoperative Anticoagulation Management in Everyday Clinical Practice: An International Comparative Analysis of Work-as-Done Using the Functional Resonance Analysis Method. J Patient Saf. 2018.

21. McNab D, Freestone J, Black C, Carson-Stevens A, Bowie P. Participatory design of an improvement intervention for the primary care management of possible sepsis using the Functional Resonance Analysis Method. BMC Med. 2018;16(1):174.

22. Clay-Williams R, Hounsgaard J, Hollnagel E. Where the rubber meets the road: using FRAM to align work-as-imagined with work-as-done when implementing clinical guidelines. Implement Sci. 2015;10:125.

23. Pickup L, Atkinson S, Hollnagel E, Bowie P, Gray S, Rawlinson S, et al. Blood sampling - Two sides to the story. Appl Ergon. 2017;59(Pt A):234-42.

24. Hollnagel E. the Functional Resonance Analysis Method FRAM Model Visualiser (FMV) 2020 [Available from: https://functionalresonance.com/the\%20fram\%20model\%20visualiser/.

25. Cheong JLY, Burnett AC, Treyvaud K, Spittle AJ. Early environment and long-term outcomes of preterm infants. J Neural Transm (Vienna). 2020;127(1):1-8.

26. Mant J. Process versus outcome indicators in the assessment of quality of health care. Int $\mathrm{J}$ Qual Health Care. 2001;13(6):475-80.

27. May CR, Johnson M, Finch T. Implementation, context and complexity. Implementation Science. 2016;11(1):141.

28. Plochg T, Klazinga NS, Starfield B. Transforming medical professionalism to fit changing health needs. BMC Med. 2009;7(1):64.

29. Purdy IB, Craig JW, Zeanah P. NICU discharge planning and beyond: recommendations for parent psychosocial support. J Perinatol. 2015;35(Suppl 1(Suppl 1):24-8.

30. Valentijn P. De kloof tussen strategie en executie van geïntegreerde zorg. Skipr. 2019;12(1):50-2.

31. Berwick DM, Nolan TW, Whittington J. The triple aim: care, health, and cost. Health Aff. 2008;27(3):759-69.

32. Catchpole K, Neyens DM, Abernathy J, Allison D, Joseph A, Reeves ST. Framework for direct observation of performance and safety in healthcare. BMJ Qual Saf. 2017;26(12):1015-21.

\section{Tables}

Due to technical limitations, tables are only available as a download in the Supplemental Files section. 
Figures

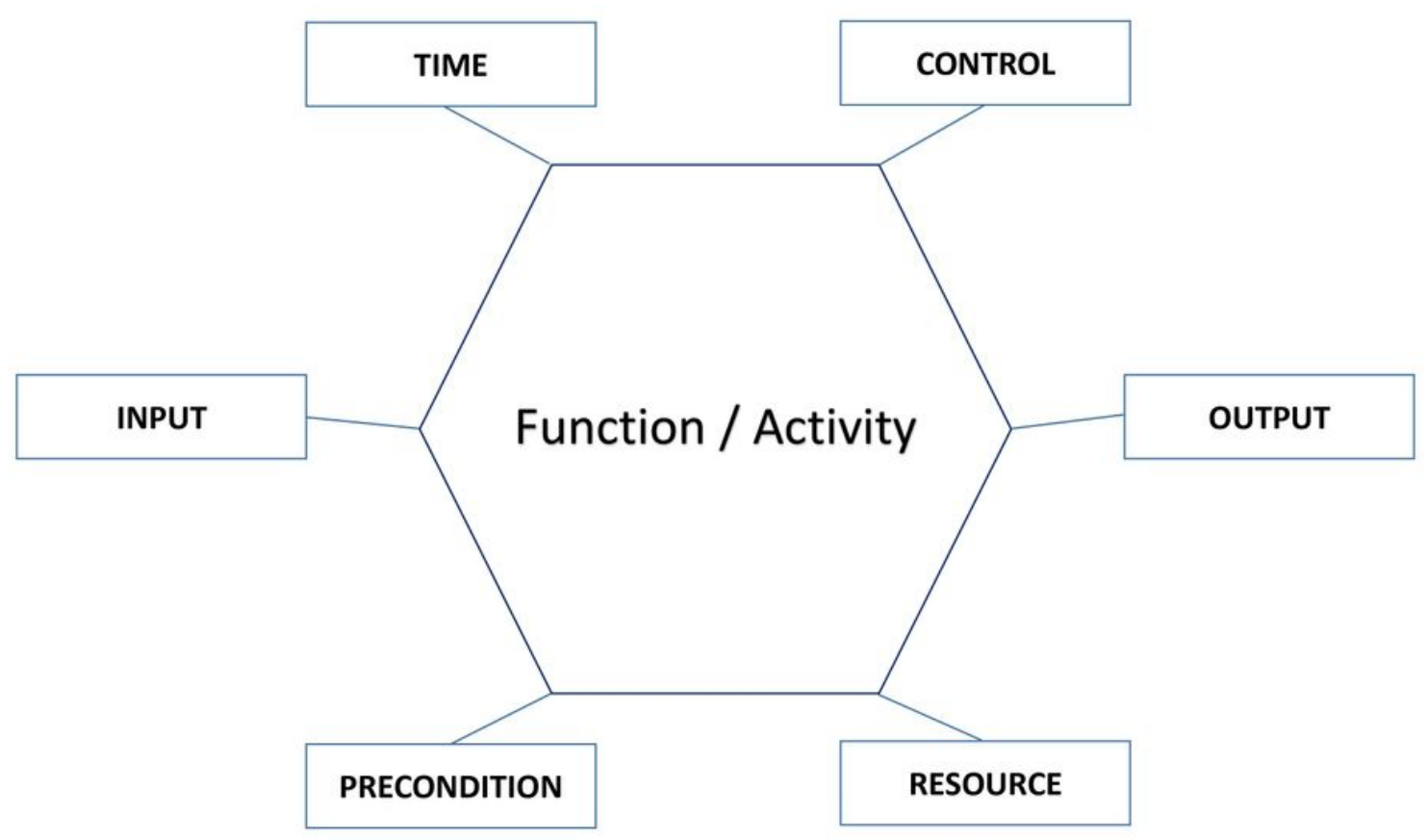

Figure 1

FRAM hexagon function with aspects 


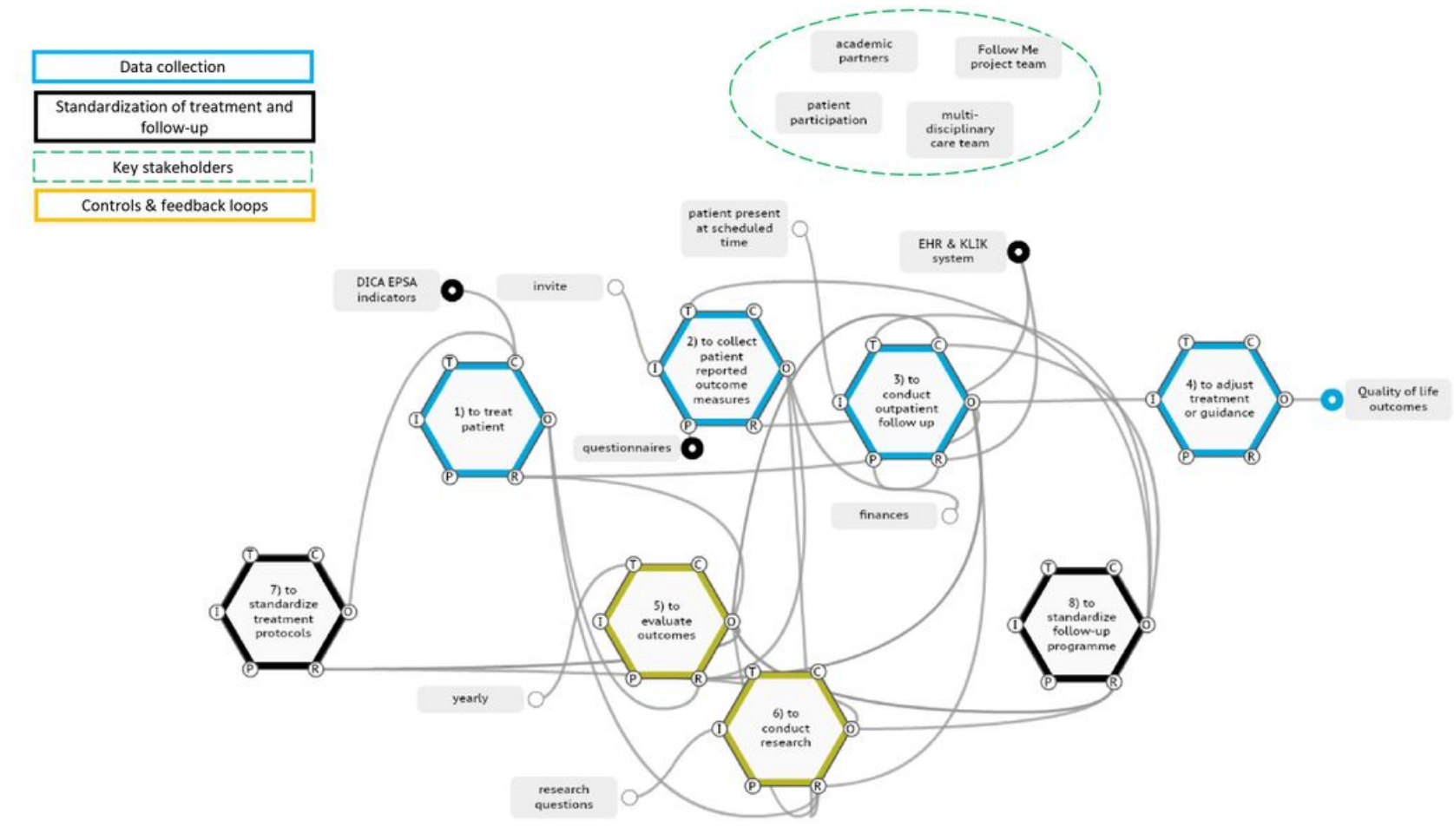

\section{Figure 2}

Work-as-imagined in Follow Me programme Functions in the work-as-imagined model of the Follow Me programme: (1) to treat patient, (2) to collect PROMs, (3) to conduct outpatient follow-up, (4) to adjust treatment or guidance, (5) to evaluate outcomes, (6) to conduct research, (7) to standardise treatment protocols, and (8) to standardise follow-up programme 


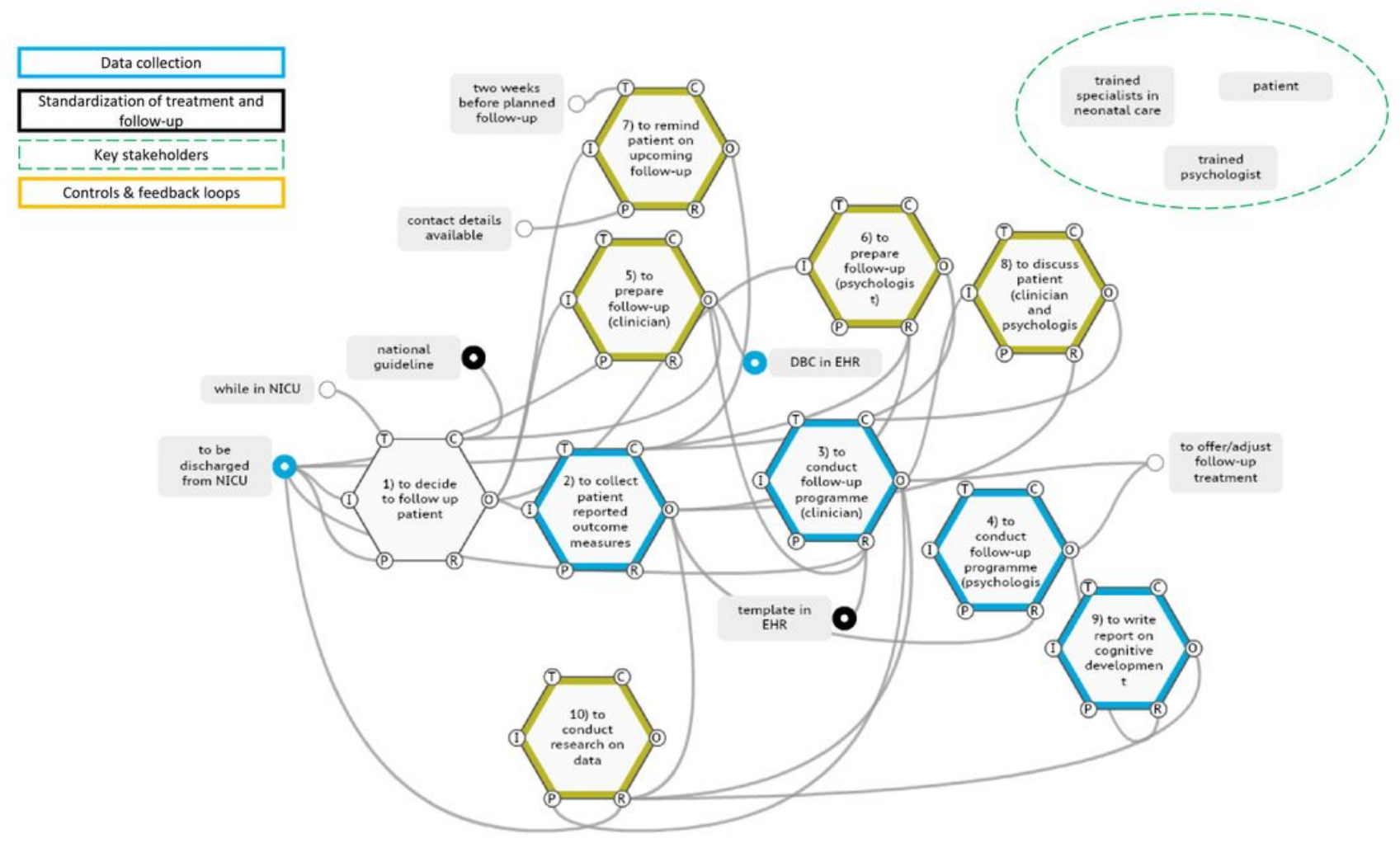

\section{Figure 3}

The work-as-done model of the AMC neonatal ICU follow-up Functions of the work-as-done model of the AMC neonatal ICU: 1) to decide to follow up patient, (2) to collect PROMs, (3) to conduct follow-up programme (clinician), (4) to conduct follow-up programme (psychologist), (5) to prepare follow-up (clinician), (6) to prepare follow-up (psychologist), (7) to remind patient of upcoming follow-up, (8) to discuss patient (clinician and psychologist), (9) to write report on cognitive development (psychologist), and (10) to conduct research on data 


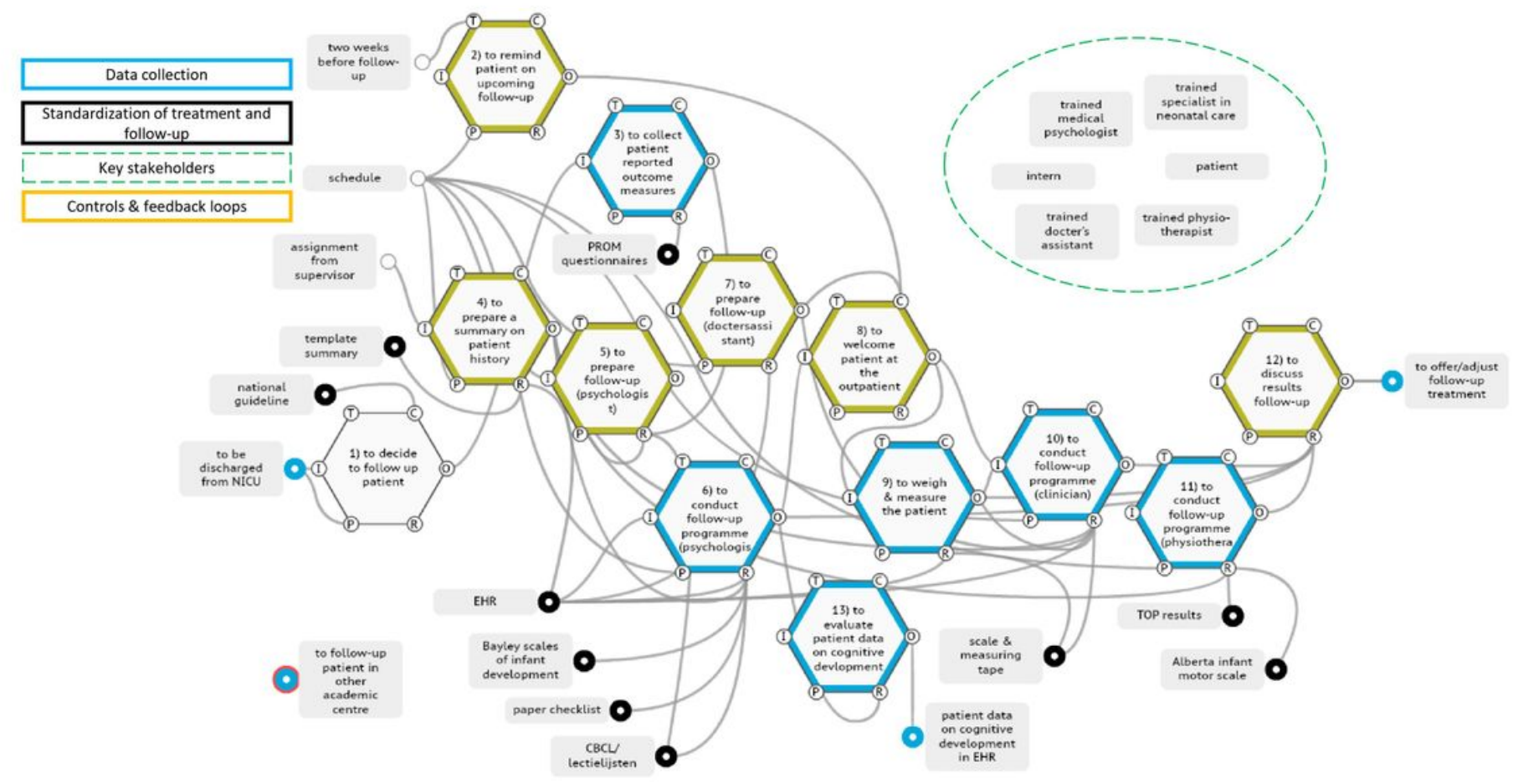

\section{Figure 4}

The work-as-done model of the VUMC neonatal ICU follow-up Functions of the work-as-done model of the VUMC neonatal ICU: (1) to decide to follow up the patient, (2) to remind patient of upcoming follow-up, (3) to collect PROMs, (4) to prepare a summary of the patient's history (psychology intern), (5) to prepare follow-up (psychologist), (6) to conduct follow-up (psychologist), (7) to prepare follow-up (medical assistant), (8) to welcome the patient at the outpatient clinic, (9) to weigh and measure the patient, (10) to conduct follow-up (neonatal specialist), (11) to conduct follow-up (physiotherapist), (12) to discuss results of follow-up, (13) to evaluate patient data on cognitive development (psychologist) 


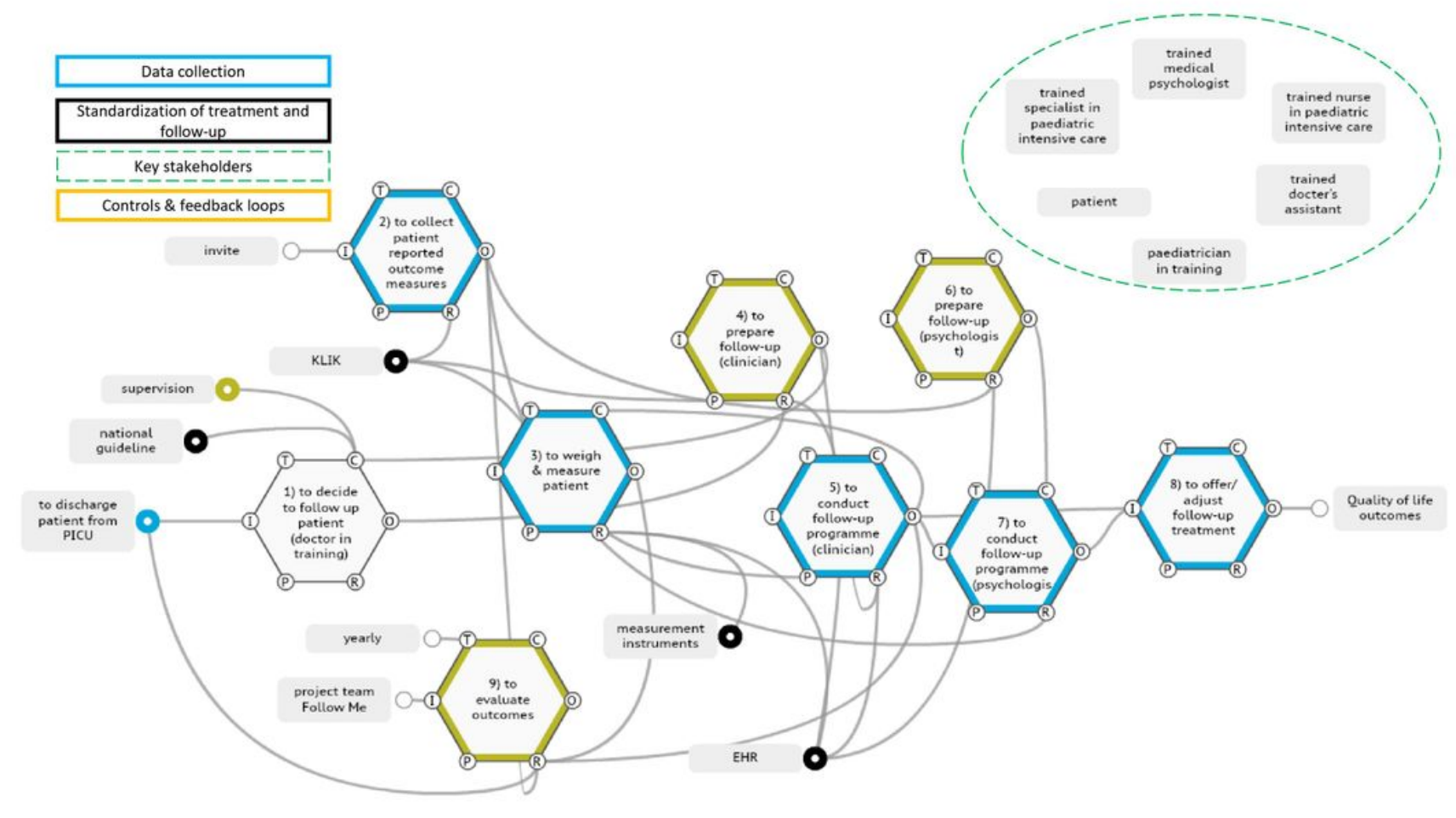

\section{Figure 5}

The work-as-done model of the paediatric ICU follow-up Functions of the work-as-done model of the paediatric ICU: (1) to decide to follow up patient (paediatrician-in-training supervised by paediatric intensivist), (2) to collect PROMs, (3) to weigh and measure patient, (4) to prepare follow-up (paediatric intensivist), (5) to conduct follow-up (paediatric intensivist), (6) to prepare follow-up (psychologist), (7) to conduct follow-up (psychologist), (8) to evaluate outcomes 


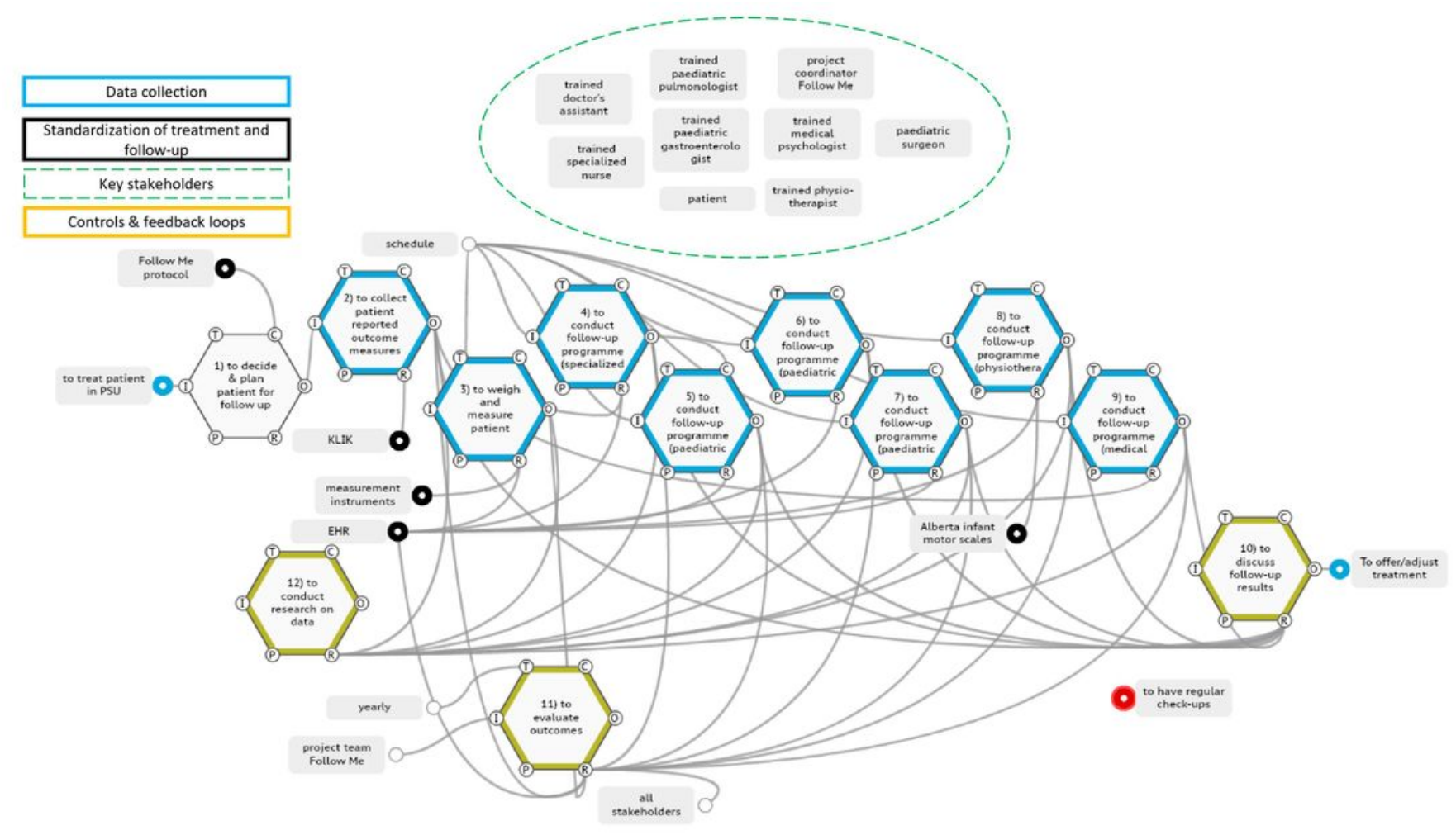

\section{Figure 6}

The work-as-done model of the paediatric surgery follow-up Functions of the work-as-done model: (1) to decide and plan patient for follow-up, (2) to collect PROMs, (3) to weigh and measure patient, (4) to conduct follow-up (clinical nurse specialist), (5) to conduct follow-up (paediatric surgeon), (6) to conduct follow-up (paediatric gastroenterologist), (7) to conduct follow-up (paediatric pulmonologist), (8) to conduct follow-up (physiotherapist), (9) to conduct follow-up (psychologist), (10) to discuss results of follow-up, (11) to evaluate outcomes, and (12) to conduct research.

\section{Supplementary Files}

This is a list of supplementary files associated with this preprint. Click to download.

- Additionalfile1COREQchecklist.pdf

- Additionalfile2TemplateObservations.pdf

- Additionalfile3Datausepermodel.pdf

- Additionalfile4Functionsgeneratingdata.pdf

- Table1.pdf

- Table2.pdf 